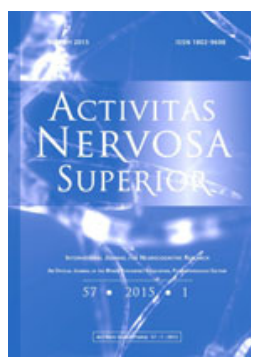

ANS: Journal for Neurocognitive Research Homepage:

www.activitas.org

\title{
CURRENT SitUATION AND CLINICAL CHARACTERISTICS OF SEDATIVE-RELATED DISORDER PATIENTS IN JAPAN: COMPARISON WITH METHAMPHETAMINE-RELATED DisORDER PATIENTS
}

\author{
Toshihiko Matsumoto" ${ }^{* 1}$, Shigeru Ozaki², Ohji Kobayashi ${ }^{3}$, Kiyoshi Wada ${ }^{1}$ \\ ${ }^{1}$ National Institute of Mental Health, National Center of Neurology and Psychiatry, Tokyo, Japan \\ 2Department of Neuropsychiatry, Tokyo metropolitan Toshima Hospital, Tokyo, Japan \\ ${ }^{3}$ Kanagawa Psychiatric Center, Yokohama, Japan
}

\begin{abstract}
The purpose of the present study is to examine the current situation of sedative-related disorders (mainly benzodiazepines) in Japan and the clinical characteristics of Japanese patients with this disorder. Subjects were 671 drug-related disorder patients diagnosed according to the ICD-10 classification as "F1: mental and behavioural disorders due to psychoactive substance use," who abused psychoactive substances other than alcohol. Of all the psychiatric hospitals in Japan between September and October 2010, these drug-related disorder patients had consecutively consulted or were admitted to 153 psychiatric hospitals. The present study was conducted by means of a mail survey. Subjects' clinical information, including history of psychoactive substance use, means of access to the primary drug of abuse, other ICD-10 diagnoses including F1 subcategory and comorbid psychiatric disorders, and recent history of self-destructive behavior, were collected from the attending psychiatrists of each subject. The data thus gathered concerning sedative-related disorder patients were compared with those patients with methamphetamine-related disorder, which has been the most serious drug-related problem in Japan since the 1950s. Out of the 671 subjects, 119 patients mainly abusing sedatives (the SRD group) were identified, while 361 patients were identified as mainly abusing methamphetamine (the MRD group). The MRD group was the largest population (53.8\% of the total subjects), followed by the SRD group $(17.7 \%)$, the inhalant-related disorder group with 56 patients $(8.3 \%)$, and so on. Compared with the MRD group, the SRD group was younger, contained more female patients, and had a lower incidence of history of involvement with anti-social societies and anti-social behavior. Patients of the SRD group were more likely to have started abusing drugs with the intention of reducing the unpleasant symptoms of insomnia (42.9\%), anxiety $(26.1 \%)$, and depression $(16.0 \%)$, and to acquire the drugs they abused from medical institutions such as psychiatric or primary care clinics $(82.1 \%)$, while patients of the MRD group were more likely to have started out of curiosity $(35.1 \%)$ or in response to peer pressure $(47.1 \%)$, and to acquire their drugs from a "pusher" (32.8\%). Additionally, in the SRD group, the ICD-10
\end{abstract}

${ }^{*}$ Correspondence to: Toshihiko Matsumoto, Department of Drug Dependence Research/Center for Suicide Prevention, National Institute of Mental Health, National Center of Neurology and Psychiatry, 4-1-1 OgawaHigashi, Kodaira, Tokyo 187-8553, Japan; Tel: +81-42-341-2711; Fax: +81-42-346-1944; email: tmatsu@ncnp.go.jp

[Translated with permission from Seishinshinkeigaku-zasshi (Psychiatria et Neurologia Japonica), vol. 113, 11841198, 2011]

Received May 16, 2013; accepted September 15, 2014; Act Nerv Super 57(1), 12-28; ISSN-1802-9698 
F1 subcategory diagnoses that were clinically most important were "dependence syndrome" (64.0\%), "harmful use" (16.2\%) and "acute intoxication" (16.2\%), while the most important subcategory diagnosis in the MRD group was "psychotic disorder" (34.3\%) and "residual disorder and late-onset psychotic disorder" (32.9\%). Further, comorbid psychiatric disorders were more frequently found in the SRD group than in the MRD group; notably, co-occurrence of mood disorder was found in $45.0 \%$ of the SRD group in contrast to the MRD group (11.9\%). Recent episodes of deliberate self-harm behavior were also more frequently found in the SRD group than the MRD group (33.6\% vs. $10.5 \%$ ); the major means by which these patients harmed themselves was by overdosing on prescribed drugs $(23.5 \% \mathrm{vs} .4 .7 \%)$. The present study suggests that sedative-related disorder is an important clinical issue in the field of drugrelated disorders in Japan today, and that SRD patients may represent a distinct type of drug abusers whose clinical characteristics are different from those of MRD patients. The development and spread of treatment programs for "dependence syndrome" and "harmful use" will help SRD patients, and educating psychiatrists about SRD will help prevent future sedative abuse.

Key words: Anxiolytics; Benzodiazepines; Drug abuse; Drug dependence; Hypnotics; Sedatives

\section{INTRODUCTION}

We have been conducting a nationwide survey of drug-related psychiatric illness in hospitals with psychiatric inpatient units (Ozaki, Wada, \& Otsuki, 2009) (referred to as Hospital Survey below) in Japan every 2 years since 1987 to determine the actual state of affairs in regard to people who abuse drugs or are dependent on drugs. The survey has been carried out using almost the same methodology each time. The Hospital Survey is the only inventory survey of patients with drug-related disorders in hospitals with psychiatric inpatient units in Japan.

Data obtained in the survey (Ozaki, Wada, \& Otsuki, 2009) have suggested that hypnotic and anxiolytic dependence and abuse in Japan are becoming major problems. The proportion of all patients with drug-related illness who have problems related to a sedative (hypnotic or anxiolytic) more than doubled in the 12 years between 1996 and 2008, and became almost as high as the proportion with problems related to organic solvents, the second most abused class of psychoactive substances after methamphetamine (Ozaki, Wada, \& Otsuki, 2009). In fact, people who work with drug addicts in the community have long recognized that increasing numbers of patients who abuse or are dependent on psychoactive substances are being admitted to rehabilitation facilities (Kondo, 2000), and they have criticized physicians who prescribe hypnotics or anxiolytics too readily (Kondo, 2010). Furthermore, psychiatry has recently come under criticism in the mass media for high-dose antipsychotic polypharmacy and for being too drugtherapy-oriented, although some reports by the mass media have been exaggerated or based on misunderstandings (Mainichi Shinbun, 2010).

The most frequently abused hypnotics and anxiolytics are benzodiazepines (BZs) and related drugs, including thienodiazepines and cyclopyrrolones (Ozaki, Wada, \& Otsuki, 2009). BZs have anti-anxiety, hypnotic, muscle-relaxant, and anticonvulsant effects, and because they are characterized by lower risks of dependence and less overdose toxicity than meprobamate and barbiturates or bromvalerylurea hypnotics, which used to be prescribed as anxiolytics, BZs have been used in psychiatric practice worldwide since the 1960s. They are prescribed by practitioners of various medical specialties in Japan.

Abuse of diazepam and other BZs and/or dependence on these drugs, however, became a matter of concern in other countries as early as the 1970s (Ribeiro et al., 2007; Rickels et al., 1983). Since the 1980s, long-term use of BZs, even within their clinical dose ranges, has been associated with physical dependency and withdrawal symptoms when discontinued (Griffiths \& Weerts, 1987; Lader \& Petursson, 1981; Rickels et al., 1983). Griffiths \& Weerts (1987) among others, have pointed out that if BZs have been used for more than 8 months, rebound insomnia/anxiety and unpleasant withdrawal symptoms outweigh the therapeutic benefit of BZs, and their long-term use may be ill-advised. In fact, long-term treatment with BZs is now disapproved of in Western countries (Tennant, \& Pumphrey, 1984). 
In reality, the amounts of BZs prescribed in Japan are much larger than in other countries. According to Murasaki (2001), in 1998-1999, the number of prescriptions for BZ anxiolytics in Japan was 6 to 20 times higher than in Western countries. Moreover, Tajima (2001) pointed out that the numbers of prescriptions of BZ anxiolytics decreased dramatically in other countries following the introduction of selective serotonin reuptake inhibitors (SSRIs) in the 1990s, but remained unchanged in Japan. However, there was no evidence of more BZ abusers or BZdependent persons in Japan than in other countries. A survey of medical institutions in the United States revealed that the two most commonly abused psychoactive substances were analgesics (pain relievers) and cannabis, and that they were followed, all at the same level, by BZs, cocaine, methamphetamine, and MDMA, which were abused by substantially smaller numbers of persons than analgesics or cannabis (Wunsch, Boyd, \& McMasters, 2009). The results of this survey suggested that there may be fewer cases of BZ abuse or dependence in other countries than in Japan because prescriptionsfor BZs are less common in other countries.

Problems associated with the prescribing of hypnotics and anxiolytics, including BZs, are not confined to drug dependence or abuse. The recent increase in number of psychiatric clinics in urban areas in Japan has been associated with an increase in the number of patients seen in emergency departments after attempting suicide by taking an overdose of psychoactive substances (Takei, 2007; Cabinets Office, 2010). In nearly $80 \%$ of cases, it has been an overdose of a BZ hypnotic or anxiolytic (Okura et al., 2008). Although overdoses of these drugs are unlikely to lead to death, they may be used for self-harm or suicide attempts, or lead to aggressive behavior in impulsive individuals due to their disinhibiting effect (The Royal College of Psychiatrists, 1997). Hirokawa et al. (2012) found that many patients who committed suicide while undergoing psychiatric treatment had taken an overdose of a BZ or other psychoactive substance immediately before engaging in highly lethal suicide behavior such as hanging. Based on these findings, they posited that the suicidal behavior of these patients may have been greatly facilitated by the state of disinhibition or intoxication induced by the overdose. In response to their findings, the Ministry of Health, Labour and Welfare of Japan instituted a study to develop measures for preventing overdoses of psychoactive substances (Ministry of health, 2009, 2010).

The clinical characteristics of Japanese patients who are dependent on prescription drugs such as hypnotics and anxiolytics have been investigated by Wada and Fukui (1991), based on data obtained in the Hospital Survey conducted in 1989. They showed that profiles of patients dependent on prescription drugs differed from profiles of those dependent on controlled substances, such as methamphetamine and organic solvents, in regard to their motivation for drug use, the person associated with their initial drug use, their personality, and their family's parenting style. Ozaki et al. (2001) divided patients whose data were reported in the Hospital Survey in 1998 into 3 groups: those with a hypnotic-related disorder (core group), those with a history of use of any illicit substance, and those who abused more than one substance. Consistent with the findings of Wada and Fukui, they found that the core group had different clinical characteristics from the other 2 groups, suggesting that the drug dependence of patients in the core group may have been iatrogenic because they were dependent on a drug that had been prescribed to treat them.

However, none of the above studies reflect the recent increase in the prevalence of hypnotic/anxiolytic-related disorders. Because of the increase in number of psychiatric clinics and outpatients (Mental health and welfare, 2010) the relevance of these studies is questionable. Moreover, the relationship between hypnotic/anxiolytic-related disorders and psychiatric care was not thoroughly investigated because the researchers did not determine how many of the patients obtained the drug they abused from a psychiatric institution or what comorbid psychiatric disorders they had. In addition, they did not investigate whether there was any association between hypnotic/anxiolytic dependence and/or abuse and suicide attempts by taking an overdose, which is a growing healthcare problem.

The objectives of the present study were to describe the current state of affairs in regard to patients with a hypnotic/anxiolytic-related disorder based on data obtained in the Hospital 
Survey in 2010, and to investigate clinical characteristics of patients with a hypnotic/ anxiolytic-related disorder compared to patients with a methamphetamine-related disorder, which is the most common form of substance-related disorder in Japan.

In this study, hypnotics and anxiolytics are collectively referred to as sedatives, including BZs and related agents such as thienodiazepines and cyclopyrrolones, and include some barbiturates and bromvalery lurea hypnotics.

\section{METHODS}

\subsection{Subjects}

The subjects of this study were patients with drug-related disorders identified in the Hospital Survey in 2010. In that year, all 1612 hospitals in Japan that had an inpatient psychiatric unit (44 national hospital organization hospitals, 139 municipal hospitals, 83 university hospitals, and 1346 private psychiatric hospitals) were asked to participate in the Hospital Survey.

Candidates for inclusion in this study were all outpatients and inpatients seen during the 2month period of September and October 2010 at the 1612 hospitals invited to participate in the survey who met the ICD-10 (World Health Organization, 1992) criteria for F1: "Mental and behavioral disorders due to use of psychoactive substances", and whose psychoactive substance of concern (primary drug responsible) was not alcohol. Responses were received from 1021 of the hospitals invited to participate (response rate: 63.3\%), and $135(8.4 \%)$ of the 1021 hospitals reported a total of 953 cases of drug-related disorders during the survey period. After excluding the 230 patients who did not consent to participate in the study and patients whose data regarding gender, age, and the primary drug responsible (described below) were missing, the remaining 671 patients (457 males and 196 females, $70.4 \%$ of all outpatients and inpatients identified during the survey period) were adopted as the subjects of this study. Their mean age (SD) was 39.5 (11.7) years, and age range was 13 to 85 years.

\subsection{Survey items}

All data used in this study were collected from the survey sheets, which contained information regarding the following:

1) Demographic variables: age and gender.

2) Episodes related to antisocial behavior: Information about past involvement with an underworld organization or juvenile gang; arrest record and/or juvenile criminal record, including arrests under the pharmaceutical affairs act; and history of incarceration in a correctional institution (e.g., juvenile classification home, juvenile training school, detention house, prison) was gleaned from "Yes" or "No" replies to questions about each of these matters.

3) Motivation for initial drug use: Subjects were asked to select one or more from the following list: enticed, thrill seeking, curiosity/interest, inability to refuse, desperation, seeking an arousal effect, relief from fatigue, seeking a sex-enhancing effect, stress reduction, relief from depression, relief from anxiety, relief from insomnia, pain relief, weight loss, and other.

4) Primary drug responsible: The attending psychiatrist of each subject was asked to select the drug or category of drug that was primarily responsible for the patient's drug-related disorder from the following list: methamphetamine, organic solvents, cannabis, sedatives (hypnotics or anxiolytics), analgesics, antitussives, multiple drugs, or other. When two or more drugs were judged to be equally responsible, the attending psychiatrist was asked to select multiple drugs.

5) Status of use in the past year and main source of the primary drug responsible: Subjects were asked about the status of use of the primary drug responsible in the past year. In addition, if the patient had used the drug during the past year, the main source of the drug 
was selectedfrom the following list (only one choice was allowed): friend, acquaintance, boyfriend/girlfriend/lover, pusher (Japanese), pusher (foreigner), psychiatrist, nonpsychiatrist physician, psychiatrist and non-psychiatrist physician, pharmacy, Internet, other, and unknown.

6) Primary ICD-10 F1 diagnosis: The primary diagnostic subcategory of drug-related disorder of each subject was selected from the F1 subcategories of the ICD-10 (only one choice was allowed).

7) ICD-10 diagnoses of comorbid psychiatric disorders: All diagnostic categories of comorbid psychiatric disorders were selected from the ICD-10 diagnostic categories (F0, F2-F9), with multiple choices allowed.

8) History of self-destructive behavior in the past year: Subjects were asked about history of self-destructive behavior, such as deliberate self-harm or suicide attempt within 1 year before the date of the survey. If any such behavior was identified, the specific means and methods of the behavior were indicated by selecting all items in the following list that applied: self-cutting (limbs), self-cutting (neck/trunk), drug use (toxic substance/controlled substance), drug use (prescription drug), hanging, jumping from a height, jumping in front of a moving vehicle, near-drowning, and other.

\subsection{Survey Methods}

The survey was conducted with the approval of the Ethics Committee of the National Center of Neurology and Psychiatry.

Prior to the survey, each hospital was sent a written explanation of the survey and was asked to post it in an appropriate place in the hospital to notify potential subjects. Attending psychiatrists collected the data by interviewing the subjects. Informed oral consent was obtained from each potential subject before the interview. If patients were capable of responding to the interview questions but refused to cooperate with the survey, they were recorded as "refusal to cooperate with the survey". No data were collected from those patients, but the attending psychiatrists were asked to report the number of suchpatients. When the interview with a subject was not possible for some other reason (e.g., patient was a minor, had already been discharged from the hospital, or their medical condition was unstable), the attending psychiatrist transcribed the relevant information for each survey item from the clinical record without obtaining informed consent from the subject. The survey sheets completed in accordance with the above procedures were sent to the first author by mail or fax, and the responses were analyzed.

\section{4. Statistical Analysis}

First, we divided the 671 patients in the study population into a group whose primary drug responsible was a sedative (sedative-related disorder [SRD] group) and a group whose primary drug responsible was methamphetamine (methamphetamine-related disorder [MRD] group). The numbers of subjects in these two groups were calculated as percentages of the entire study population, and the variables related to survey items were compared between the two groups.

All statistical analyses were performed using the SPSS ver. 17.0 for Windows software program. We used Pearson's $\chi^{2}$ test for comparisons of qualitative variables andStudent's $t$ test for comparisons of quantitative variables (e.g., age). All analyses were performed as twotailed tests with a significance level of $5 \%$. However, because multiple comparisons between the SRD group and MRD group were made for each survey item, it was necessary to avoid Type II errors by adjusting the P-values obtained by Bonferroni correction (the P-value obtained is multiplied by $n$, where $n$ is the number of comparisons being made for the survey item). Results were concluded to be significant when the corrected P-value was less than 0.05 . 


\section{RESULTS}

3.1. Numbers of subjects in the SRD group and MRD group as percentages of the entire study population

The 671 subjects were classified according to their primarydrug responsible into the following groups: methamphetamine, 361 (53.8\%); sedative (hypnotic or anxiolytic), 119 (17.7\%); multiple-drug, 57 (8.5\%); organic solvent, 56 (8.3\%); antitussive, 20 (3.0\%); cannabis, 18 (2.7\%); analgesic, 12 (1.8\%); and otherdrug, $28(4.2 \%)$.

Thus, there were 119 subjects (56 males and 63 females) in the SRD group and 361 subjects in the MRD group (268 males and 93 females). The SRD group contained a higher percentage of females than the MRD group $(\mathrm{P}<0.001)$. Mean age (SD) was 38.0 (13.1) years in the SRD group and 41.4 (11.3) years in the MRD group, with the mean age of the SRD group significantly lower than of the MRD group $(\mathrm{P}=0.008)$.

The relatively commonly reported sedative drugs in the write-in part of the survey by number of subjects were flunitrazepam (40), etizolam (32), triazolam (28), zolpidem (22), diazepam (10), alprazolam (9), nitrazepam (6), brotizolam (5), and bromvalerylurea (4). Most of them were BZs or related substances.

\subsection{Episodes of antisocial behavior}

Table 1 compares episodes of antisocial behavior in the SRD group and MRD group. As shown in the table, significantly lower proportions of subjects in the SRD group reportedpast involvement with an underworldorganization, past involvement with a juvenile gang, arrestor juvenile criminal record, or history of incarceration in a correctional institution compared to the MRD group.

Table 1. Comparison between episodes of antisocial behavior in the SRD group and MRD group.

\begin{tabular}{lccccc}
\hline & \multicolumn{2}{l}{ SRD group } & \multicolumn{2}{l}{ MRD group } \\
& \multicolumn{2}{l}{$\mathrm{N}=119$} & \multicolumn{2}{c}{$\mathrm{N}=361$} & \\
& $\mathrm{~N}$ & $\%$ & $\mathrm{n}$ & $\%$ & $\chi^{2}$ \\
\hline Involvement with an underworld organization* & 33 & $27.7 \%$ & 266 & $73.7 \%$ & 80.459 \\
Involvement with a juvenile gang* & 40 & $33.6 \%$ & 269 & $74.5 \%$ & 65.287 \\
Arrest record and/or juvenile criminal record* & 33 & $27.7 \%$ & 265 & $73.4 \%$ & 79.321 \\
Incarceration in a correctional institution* & 14 & $11.8 \%$ & 243 & $67.3 \%$ & 111.019 \\
\hline
\end{tabular}

Note. $* \mathrm{P}<0.05$ (with Bonferroni correction)

\subsection{Motivation for initial drug use}

In Table 2 is compared motivation for initial drug use in the SRD group and MRD group. Significantly higher proportions of subjects in the SRD group than in the MRD group stated that their motivation for initial drug use was to obtain relief from insomnia ( $42.9 \%$ vs. $0.6 \%)$, relief from anxiety $(26.1 \%$ vs. $2.0 \%)$, relief from depression $(16.0 \%$ vs. $2.6 \%)$, or desperation $(16.0 \%$ vs. $3.4 \%)$. However, a significantly higher proportion of subjects in the MRD group 
compared to the SRD group stated that their motivation for initial drug use was curiosity/interest ( $35.1 \%$ vs. $7.6 \%)$.

\section{4. Source of the drug}

Use of the primarydrug responsible in the past year was admitted by 109 subjects (91.6\%) in the SRD group and by 177 subjects (49.0\%) in the MRD group. Table 3 shows the sources from whichthey obtained the primarydrug responsible usedwithin the past year.

Significantly higher proportions of subjects in the SRD group than in the MRD groupobtained the drug from a psychiatrist $(60.0 \%$ vs. $0.0 \%)$, psychiatrist and non-psychiatrist physician ( $15.0 \%$ vs. $0.0 \%)$, pharmacy (7.3\% vs. $0.0 \%)$, ornon-psychiatrist physician $(7.3 \%$ vs. $0.0 \%)$.

By contrast, significantly higher proportions of subjects in the MRD group than in the SRD group responded thatthe source of the drugwas unknown $(33.9 \%$ vs. $0.9 \%)$ or a Japanese pusher $(32.9 \%$ vs. $0.9 \%)$.

Table 2. Comparison between motivations for initial drug use in the SRD group and MRD group (Multiple choices allowed).

\begin{tabular}{|c|c|c|c|c|c|}
\hline & \multicolumn{2}{|c|}{$\begin{array}{c}\text { SRD group } \\
\mathrm{N}=119\end{array}$} & \multicolumn{2}{|c|}{$\begin{array}{c}\text { MRD group } \\
\mathrm{N}=361\end{array}$} & \multirow[b]{2}{*}{$\chi^{2}$} \\
\hline & $\mathbf{n}$ & $\%$ & $\mathbf{n}$ & $\%$ & \\
\hline Enticed* & 9 & $7.6 \%$ & 165 & $47.1 \%$ & 55.085 \\
\hline Thrill seeking* & 2 & $1.7 \%$ & 56 & $16.0 \%$ & 15.698 \\
\hline Curiosity/interest* & 9 & $7.6 \%$ & 123 & $35.1 \%$ & 30.614 \\
\hline Inability to refuse & 1 & $0.8 \%$ & 23 & $6.6 \%$ & 5.257 \\
\hline Desperation* & 19 & $16.0 \%$ & 12 & $3.4 \%$ & 26.400 \\
\hline Seeking an arousal effect & 2 & $1.7 \%$ & 12 & $3.4 \%$ & 0.795 \\
\hline Relief from fatigue & 5 & $4.2 \%$ & 12 & $3.4 \%$ & 0.244 \\
\hline Seeking a sex-enhancing effect & 2 & $1.7 \%$ & 13 & $3.7 \%$ & 1.023 \\
\hline Stress reduction & 8 & $6.7 \%$ & 23 & $6.6 \%$ & 0.037 \\
\hline Relief from depression * & 19 & $16.0 \%$ & 9 & $2.6 \%$ & 30.554 \\
\hline Relief from anxiety* & 31 & $26.1 \%$ & 7 & $2.0 \%$ & 73.412 \\
\hline Relief from insomnia* & 51 & $42.9 \%$ & 2 & $0.6 \%$ & 167.309 \\
\hline Pain relief & 2 & $1.7 \%$ & 3 & $0.9 \%$ & 0.670 \\
\hline Weight loss & 2 & $1.7 \%$ & 3 & $0.9 \%$ & 0.670 \\
\hline Other & 6 & $5.0 \%$ & 7 & $2.0 \%$ & 3.444 \\
\hline
\end{tabular}

Note. $* \mathrm{P}<0.05$ (with Bonferroni correction) 


\subsection{Primary ICD-10 F1 subcategory diagnosis}

Data in relation to the primary ICD-10 F1 subcategory diagnosis for some patients were missing. Thus, only 111 patients $(93.3 \%)$ in the SRD group and $350(97.0 \%)$ in the MRD group weresubjects of the analysis of this item. Table 4 compares the results for the primary F1 subcategory diagnosis of patientsin the SRD group and MRD group. The subcategory diagnoses Dependence syndrome (64.0\% vs. $25.7 \%)$, Harmful use $(16.2 \%$ vs. $1.7 \%)$, and Acute intoxication $(16.2 \%$ vs. $2.6 \%)$ were significantly more common in the SRD group than in the MRD group. Psychotic disorder $(34.3 \%$ vs. $0.0 \%)$ and Residual disorder and late-onset psychotic disorder (32.9\% vs. $0.9 \%$ ) were significantly more common in the MRD group than in the SRD group.

Table 3. Comparison between sources of the primary drug responsible in the SRD group and MRD group ( $\mathrm{N}=$ patients who had used the drug within the past year; multiple choices allowed).

\begin{tabular}{lccccc}
\hline & \multicolumn{3}{l}{ SRD group } & \multicolumn{3}{l}{ MRD group } \\
& \multicolumn{2}{l}{$\mathrm{N}=109$} & \multicolumn{2}{l}{$\mathrm{N}=177$} \\
& $\mathrm{n}$ & $\%$ & $\mathrm{n}$ & $\%$ & $\chi^{2}$ \\
\hline Friend & 5 & $4.6 \%$ & 18 & $10.2 \%$ & 0.156 \\
Acquaintance & 1 & $0.9 \%$ & 23 & $13.0 \%$ & 5.961 \\
Boyfriend/girlfriend/lover & 0 & $0.0 \%$ & 7 & $4.0 \%$ & 2.399 \\
Family & 2 & $1.8 \%$ & 3 & $1.7 \%$ & 0.585 \\
Pusher (Japanese)* & 1 & $0.9 \%$ & 58 & $32.8 \%$ & 19.943 \\
Pusher (foreigner) & 0 & $0.0 \%$ & 6 & $3.4 \%$ & 2.051 \\
Psychiatrist* & 65 & $60.0 \%$ & 0 & $0.0 \%$ & 225.103 \\
Non-psychiatrist physician * & 8 & $7.1 \%$ & 0 & $0.0 \%$ & 24.148 \\
Psychiatrist and non-psychiatrist physician * & 17 & $15.0 \%$ & 0 & $0.0 \%$ & 52.386 \\
Pharmacy* & 8 & $7.3 \%$ & 0 & $0.0 \%$ & 24.148 \\
Internet & 0 & $0.0 \%$ & 2 & $1.1 \%$ & 0.678 \\
Other & 1 & $0.9 \%$ & 0 & $0.0 \%$ & 2.097 \\
Unknown* & 1 & $0.9 \%$ & 60 & $33.9 \%$ & 20.824 \\
\hline
\end{tabular}

Note. ${ }^{*} \mathrm{P}<0.05$ (with Bonferroni correction)

\subsection{Comorbid psychiatric disorders}

In the Table 5 are compared ICD-10 diagnoses of comorbid psychiatric disorders in the SRD group and MRD group. Higher proportions of patients in the SRD group than in the MRD group were diagnosedwith a comorbid psychiatricdisorderin F3 (45.0\% vs. $11.9 \%)$, F6 (25.2\% vs. $11.6 \%)$, F5 (17.6\% vs. $1.1 \%)$, or F4 (17.6\% vs. $6.9 \%)$. In contrast, no diagnostic categories were significantly more commonly diagnosed in the MRD group than in the SRD group. 
Table 4. Comparison between primary ICD-10 F1 diagnoses in the SRD group and MRD group (Single choice).

\begin{tabular}{lccccc}
\hline & \multicolumn{2}{c}{ SRD group } & \multicolumn{2}{c}{ MRD group } \\
& \multicolumn{2}{c}{$\mathrm{N}=111$} & \multicolumn{2}{c}{$\mathrm{N}=350$} & \\
& $\mathrm{n}$ & $\%$ & $\mathrm{n}$ & $\%$ & $\chi^{2}$ \\
\hline Acute intoxication* $^{*}$ & 18 & $16.2 \%$ & 9 & $2.6 \%$ & 25.297 \\
Harmful use $^{*}$ & 18 & $16.2 \%$ & 6 & $1.7 \%$ & 32.214 \\
Dependence syndrome $^{*}$ & 71 & $64.0 \%$ & 90 & $25.7 \%$ & 44.576 \\
Withdrawal state $^{*}$ & 1 & $9.0 \%$ & 1 & $0.3 \%$ & 0.621 \\
Withdrawal state with delirium $^{*}$ & 0 & $0.0 \%$ & 0 & $0.0 \%$ & - \\
Psychotic disorder* $^{*}$ & 0 & $0.0 \%$ & 120 & $34.3 \%$ & 54.632 \\
Amnesic syndrome & 0 & $0.0 \%$ & 11 & $0.3 \%$ & 0.347 \\
Residual and late-onset psychotic disorder* & 1 & $0.9 \%$ & 115 & $32.9 \%$ & 48.854 \\
Other mental and behavioural disorders & 2 & $1.8 \%$ & 8 & $2.3 \%$ & 0.171 \\
\hline
\end{tabular}

Note. $* \mathrm{P}<0.05$ (with Bonferroni correction)

Table 5. Comparison between comorbid psychiatric disorders [diagnoses] in the SRD group and MRD group (Multiple choices allowed).

\begin{tabular}{|c|c|c|c|c|c|}
\hline & \multirow{2}{*}{\multicolumn{2}{|c|}{$\begin{array}{l}\text { SRD group } \\
\mathrm{N}=111\end{array}$}} & \multirow{2}{*}{\multicolumn{2}{|c|}{$\begin{array}{l}\text { MRD group } \\
\mathrm{N}=350\end{array}$}} & \multirow[b]{3}{*}{$\chi^{2}$} \\
\hline & & & & & \\
\hline & $\mathbf{n}$ & $\%$ & $\mathrm{n}$ & $\%$ & \\
\hline $\begin{array}{l}\text { F0 Organic, including symptomatic, mental } \\
\text { disorders }\end{array}$ & 1 & $0.8 \%$ & 6 & $1.7 \%$ & 0.421 \\
\hline $\begin{array}{l}\text { F2 Schizophrenia, schizotypal and delusional } \\
\text { disorders }\end{array}$ & 4 & $3.4 \%$ & 39 & $10.8 \%$ & 6.077 \\
\hline F3 Mood (affective) disorders* & 45 & $45.0 \%$ & 43 & $11.9 \%$ & 40.110 \\
\hline $\begin{array}{l}\text { F4 Neurotic, stress-related and somatoform } \\
\text { disorders* }\end{array}$ & 21 & $17.6 \%$ & 25 & $6.9 \%$ & 11.874 \\
\hline $\begin{array}{l}\text { F5 Behavioural syndromes associated with } \\
\text { physiological disturbances and physical } \\
\text { factors* }\end{array}$ & 21 & $17.6 \%$ & 4 & $1.1 \%$ & 49.587 \\
\hline $\begin{array}{l}\text { F6 Disorders of adult personality and } \\
\text { behaviour* }\end{array}$ & 30 & $25.2 \%$ & 42 & $11.6 \%$ & 12.937 \\
\hline F7 Mental retardation & 2 & $1.7 \%$ & 11 & $3.0 \%$ & 0.634 \\
\hline F8 Disorders of psychological development & 4 & $3.4 \%$ & 2 & $0.5 \%$ & 5.714 \\
\hline $\begin{array}{l}\text { F9 Behavioural and emotional disorders with } \\
\text { onset usually occurring in childhood and } \\
\text { adolescence }\end{array}$ & 0 & $0.0 \%$ & 1 & $0.3 \%$ & 0.330 \\
\hline
\end{tabular}

Note. ${ }^{*} \mathrm{P}<0.05$ (with Bonferroni correction) 
Table 6. Comparison between self-destructive behaviors in the past year in the SRD group and MRD group.

\begin{tabular}{|c|c|c|c|c|c|}
\hline & \multicolumn{2}{|c|}{$\begin{array}{c}\text { SRD group } \\
\mathrm{N}=119\end{array}$} & \multicolumn{2}{|c|}{$\begin{array}{l}\text { MRD group } \\
\qquad \mathrm{N}=361\end{array}$} & \multirow[b]{2}{*}{$\chi^{2}$} \\
\hline & $\mathrm{n}$ & $\%$ & $\mathbf{n}$ & $\%$ & \\
\hline $\begin{array}{l}\text { Self-destructive behaviors in the } \\
\text { past year* }\end{array}$ & 40 & $33.6 \%$ & 38 & $10.5 \%$ & 35.052 \\
\hline Self-cutting (limbs) & 14 & $11.7 \%$ & 18 & $5.0 \%$ & 6.609 \\
\hline Self-cutting (neck/trunk) & 3 & $2.5 \%$ & 4 & $1.1 \%$ & 1.246 \\
\hline $\begin{array}{l}\text { Drug use (toxic/controlled } \\
\text { substance) }\end{array}$ & 5 & $4.2 \%$ & 4 & $1.1 \%$ & 4.656 \\
\hline Drug use (prescription drug) * & 28 & $23.5 \%$ & 17 & $4.7 \%$ & 37.312 \\
\hline Hanging & 3 & $2.5 \%$ & 8 & $2.2 \%$ & 0.037 \\
\hline Jumping from a high place & 3 & $2.5 \%$ & 6 & $1.7 \%$ & 0.359 \\
\hline $\begin{array}{l}\text { Jumping in front of a moving } \\
\text { vehicle }\end{array}$ & 1 & $0.8 \%$ & 3 & $0.8 \%$ & 0.000 \\
\hline Near-drowning & 0 & $0.0 \%$ & 0 & $0.0 \%$ & - \\
\hline Other & 3 & $2.5 \%$ & 6 & $1.7 \%$ & 0.359 \\
\hline
\end{tabular}

Note. ${ }^{*} \mathrm{P}<0.05$ (with Bonferroni correction)

Figure 1. Changes over time in the proportions (\%) of drug-related disorder patients according to the primary drug responsible.

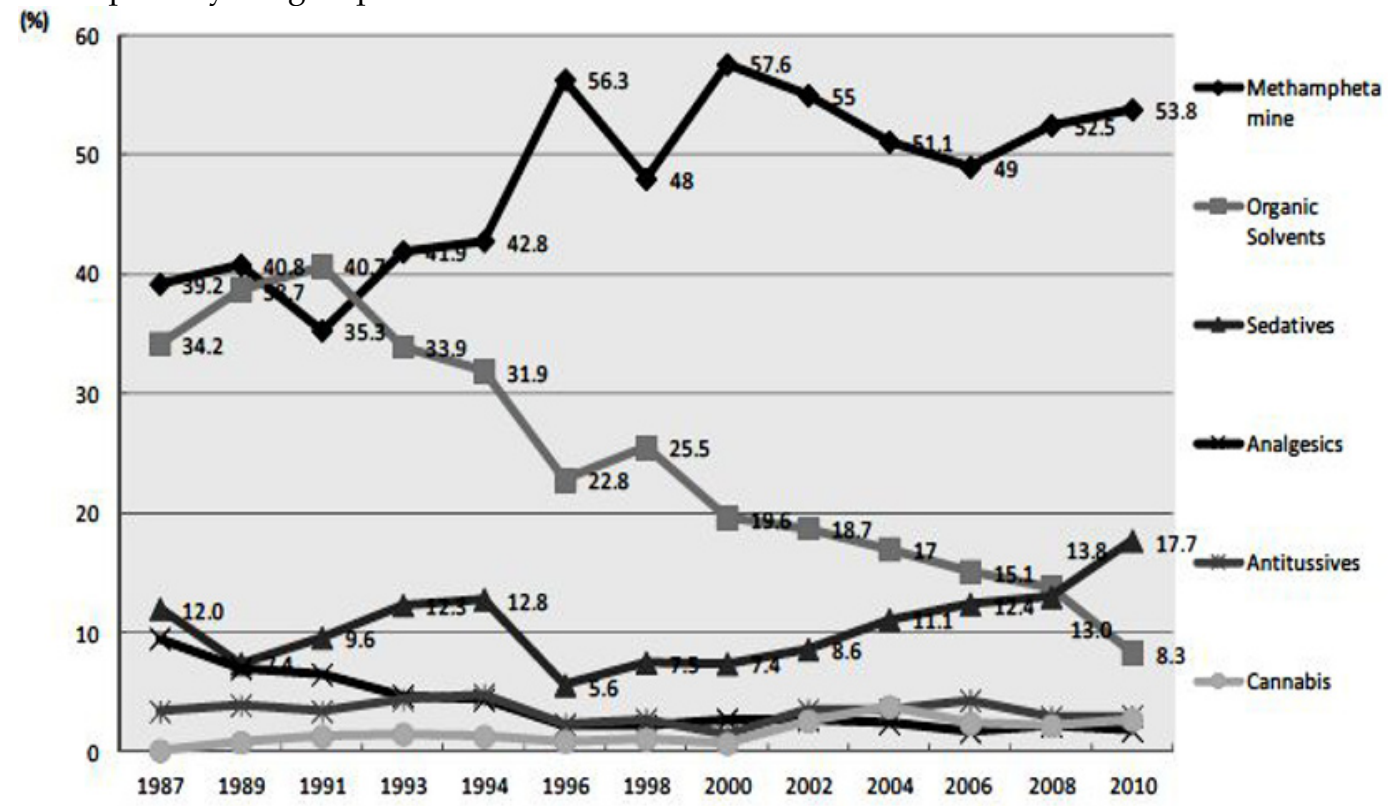




\subsection{Self-destructive behavior}

Self-destructive behavior in the past year in the SRD group and MRD group is compared in Table 6. A significantly higher proportion of subjects in the SRD group than in the MRD group had engaged in self-destructive behavior $(33.6 \%$ vs. $10.5 \%)$. A comparison of specific means/methods of self-destructive behavior showed that drug use (prescription drugs) was significantly more common in the SRD group than in the MRD group ( $23.5 \%$ vs. $4.7 \%)$.

\section{DISCUSSION}

The Hospital Survey conducted in 2010 is the most recent survey available that reveals the current situation in regard to sedative-related disorders in Japan based on a nationwidesample. The study reported here is the only one that has investigated the clinical characteristics of patients with sedative-related disorders compared to those of patients with psychiatric disorders related to methamphetamine, which is the most commonly abused drug in Japan.

The results of the present study, focusing on the current situation and trends in sedativerelated disorders in Japan, as well as their clinical characteristics, are discussed below.

\subsection{Trends in sedative-related disorders}

We found that $119(17.7 \%)$ of the 671 patients with a drug-related disorder surveyed in this study had problems related to sedatives, which were the second most primary drugs responsible, after methamphetamine. This means that sedatives had replaced organic solvents, which had been the second most abused drug after methamphetamine, since the first survey in 1987.

Figure 1 shows changes over time in the proportions (\%) of subjects who identified each substance as being the primary drug responsible for their drug-related disorder in this series of surveys. It is impossible to make simple comparisons because each survey was different in terms of response rates and total numbers of cases in the institutions surveyed. However, data reveal that the proportion of cases in which an organic solvent was considered the primary drug responsible has declined steadily and sharply since 1993, whereas the percentage of cases in which the primary drug responsible was considered a sedative gradually increased over the same period. By the time of the previous survey in 2008 (Ozaki, Wada, \& Otsuki, 2009), the gap between the proportions of cases in which the primary drug responsible was an organicsolvent $(15.1 \%)$ compared to those in which it was a sedative $(12.4 \%)$ had already narrowed substantially.

In view of the above findings, the increase in proportion of SRD patients observed in this survey may be related to the decreasein number of patients whose primary drug responsible was an organic solvent. Possible collateral evidence is provided by a nation wide survey on awareness and the current status of drinking/smoking/drug abuse by junior-high school students in Japan (Wada et al., 2011), which was conducted concurrently with this study and found that the percentage of junior-high school students with lifetime experience of organic solvent use had decreased.

Nonetheless, the fact that sedatives became the second most abused category of drugs has significant implications forfuture clinical practice in regard to drug-related disorders in Japan. The most abused drugs in Japan used to be controlled substances, such as methamphetamine and organic solvents, whose very useconstitutes a criminal act. For that reason, the methamphetamine dependence that persisted after a drug-induced psychotic disorder had resolved could be treated as a judicial problem and excluded from medical care. However, as the results of this study show, an increasing number of patients with a drug-related disorder 
that cannot be settled in a judicial setting have increased over time as a proportion of drugabuse patients seen in clinical practice settings in Japan. In that sense, as pointed out in a previous study (Wada, 2011), it seems fair to say that psychiatric care today cannot avoid accepting "inability to use hypnotics or anxiolytics appropriately" per se as an indication for medical care.

\subsection{Clinical characteristics of patients with a sedative-related disorder}

The results of this study showed that various aspects of the clinical characteristics of SRD patients differ from those of patients with a MRD, which has consistently been the primary problem inclinical practice in regard to drug-related disorders in Japan. The SRD group in this study was on the whole younger and contained a higher proportion of female patients compared to the MRD group. In addition, subjects in the SRD group were much less likely to have had a history of involvement with an under world organization or juvenile gang or a record of arrestor juvenile crime. These findings appear to reflect the fact that, unlike methamphetamine, sedatives are not illicit substances that are encountered through contact with antisocial groups.

The differences between motivations for initial drug use reported by SRD patients and MRD patients were interesting. MRD patients were more likely to have started using the drugbecause of being enticed or because of curiosity/interest or thrill seeking, whereas larger proportions of SRD patients reported that their motivation to start using the drug was to obtain relief from insomnia, anxiety, or depression, or out of desperation. These findings suggest that sedatives may have initially been used as a regular medical treatment to reduce distress, for example, or as a kind of inappropriate self-medication (Khantzian, 1990), in contrast to methamphetamine, which most patients started to use because of peer pressure or for thrills or pleasure.

The findings described here are consistent with findings reported in previous studies conducted in Japan. Wada and Fukui (1991) and Ozaki et al. (2001) reported that SRD patients are different from patients with controlled-drug-related disorders, in that few of them have connections with a juvenile gang or underworld organization, and they rarely have a record of arrestor juvenile crime or a history of incarceration in a correctional institution. Moreover, Wada and Fukui (1991) showed that patients who are dependent on a prescription drug or abuse a prescription drug, such as a hypnotic or anxiolytic, havecharacteristics that distinguish them from methamphetamine/organic solvent addicts or abusers. The former were more likely to start using the drug toobtain relief from psychological or physical distress, whereas the motivation for initial drug use by the latter was more likely to be curiosity or enticement by a peer. Thus, the clinical characteristics of SRD patients appear to have remained essentially unchanged over the past 20 years.

The results of this study also confirmed marked differences in the primary F1 subcategory diagnoses in the ICD-10 between SRD and MRD patients. More specifically, while MRD patients were more likely to be diagnosed with Psychotic disorder or Residual disorder and late-onset psychotic disorder, the diagnoses of the SRD patients were more likely to be Dependence syndrome, Harmful use, or Acute intoxication. Thus, the most important clinical task in relation to MRD patients is to resolve their psychotic symptoms, whereas the inability to use drugs appropriately is the primary problem to be resolved in SRD patients. Although a smaller number of the MRD patients whose primary diagnosis was a psychotic symptom presumably also had Dependence syndrome or Harmful use as a secondary diagnosis, the fact thata considerably higher proportion of patients in the SRD group compared to the MRD group had used the drug in the past year (91.6\% vs. $49.0 \%)$ cannot be ignored.

These results are not surprising in view of the pharmacologic actions of each of the drugs abused because sedatives differ from methamphetamine in terms of not possessing psychogenic properties and instead having an inhibitory effect on the central nervous system that has cross tolerance with alcohol. As a result, the treatment of sedative-related disorders 
inevitably requires dealing with the problem of drug use from the beginning. This is in contrast to the treatment of methamphetamine-related disorders, which requires treatment of the psychosis first. A vicious cycle may very well exist in which the fact that sedatives are not legally regulated encourages the medicalization of drug use disorders, and the need to treat comorbid psychiatric disorders discussed below allows continued use of the abused drug, and in turn increases the need for treatment of drug use disorders. In any event, as sedativerelated disorders increase as a proportion of drug-related disorders in the future, it is highly probable that the focus of clinical practice in regard to drug-related disorders will shift from the treatment of drug-induced psychosis to the treatment of drug use disorders.

In view of such expectations for the future, the fact that only a few medical institutions in Japan can properly treat drug dependence is a concern. Japanese psychiatric professionals tend to have strong aversive feelings toward drug-related disorders in the first place, and only a very small number of hospitals accept such patients (only $8.4 \%$ [n=135] of the 1021 hospitals that participated in this survey reported treating outpatients and inpatients with drug-related disorders). Moreover, even fewer institutions provide programs specifically designed for the treatment of drug dependence (Ozaki, Wada, \& Otsuki, 2009). Even institutions that specialize in such treatment often use programs designed for alcohol addiction as substitutes and send patients to non-medical, private rehabilitation facilities as soon as they recover from a druginduced psychosis or acute intoxication.

There is an urgent need to develop and make drug dependence treatment programs widely available in psychiatric institutions in order to establish a treatment system for sedativerelated disorders. More specifically, the SMARPP (Serigaya Methamphetamine Relapse Prevention Program; Matsumoto \& Kobayashi, 2010), a comprehensive treatment program for drug dependence based on workbooks and manuals, is a candidate program for dissemination to psychiatric institutions nationwide. SMARPP was originally developed for the treatment of methamphetamine abuse/dependence, but is now being applied to the treatment of abuse and dependence of a variety of substances, including sedatives and alcohol. We have conducted SMARPP through research projects and workshops throughout Japan.

\subsection{Challenges of psychiatric care for sedative-related disorders in Japan}

The results of this study suggest that psychiatric care itself may have promoted the development of sedative-related disorders. More specifically, the drug source reported by MRD patients who had used methamphetamine in the past year was more likely tohave been a Japanese pusher or to be unknown (there are limits to the ability to collect information regarding sources of controlled substances from patients who are in clinical settings). However, SRD patients often reported health care professionals, including a psychiatrist, nonpsychiatrist physician, or pharmacy as their drug source.It is particularly noteworthy that a psychiatristwas the drug source for $75 \%$ of the SRD patients.

The relationship between sedative-related disorders and psychiatrists appears to have become even stronger in recent years. The Hospital Survey in 1989 (Ozaki, Wada, \& Otsuki, 2009) compiled statistics separately for hypnotics and anxiolytics and showed that $35.3 \%$ and $47.1 \%$ of patients who abused or were addicted to a hypnotic or an anxiolytic, respectively, reported having obtained the drug from a physician (at that timeno distinction was made between psychiatrists and other physicians). These percentages, however, were far lower than percentages inthe present study.

It may be unreasonable to blame psychiatrists for prescribing patients drugs that are abused. In this study, 45\% of SRD patients had the ICD-10 psychiatric comorbidity F3: Mood (affective) disorders and many patients were identified as having comorbid F6: Disorders of adult personality and behavior, F4: Neurotic, stress-related and somatoform disorders, and/or F5: Behavioral syndromes associated with physiological disturbances and physical factors. These results are consistent with findings reported by previous studies in other countries (Ribeiro et al., 2007; Tennant \& Pumphrey, 1984). As Khantzian (1990) claims, there is also the 
possibility that sedative dependence/abuseis "inappropriate self-medication" for a psychiatric disorder that the patient had developed previously. In such cases, attending psychiatrists sometimes have no choice but to continue the pharmacotherapy targeted at the concomitant psychiatric disorder even though they are aware of the patient's sedative abuse or dependence. Thus, psychiatrists may have becomethe "drug source" against their will.

Nevertheless, it is very risky to routinely continue to prescribe sedatives for SRD patients with comorbidities in the form of depressive disorder (Lönnqvist et al., 1995) or a personality disorder (Linehan, Shireen, \& Welch, 2006; Takei et al., 2007), which are risk factors for suicide. In fact, in the present study we found that a higher proportion of SRD patients compared to MRD patients had engaged in self-harm or a suicide attempt, and that the method used for such self-destructive behavior was more likely to be use of prescription drugs, presumably an overdose of a sedative. This means that SRD patients are not simply drug abusers or addicts, but a group of people who are at high risk of suicidal behavior, suggesting a possible overlapbetween the populations of sedative dependence/abuse patients and overdose patients.

Because data related to suicidal behavior were not collected in the Hospital Survey conducted in 1989 (Ozaki, Wada, \& Otsuki, 2009) or 1998 (Ozaki, S., Kikuchi, \& Wada, 2001), it is impossible to know whether the suicidal tendencies and affinity for overdosing by SRD patients are specific to recent years or already existed at the time of the Hospital Survey that served as the basis for the present study. However, in view of the lower psychological resistance to consulting psychiatric services these days and the increasing numbers of psychiatric clinics and outpatients from year to year (Mental health and welfare, 2010), more and more people in Japancan be assumed to have taken sedatives. In addition, the regulation that limited the number of daysa psychoactive substance could be prescribed was relaxed in 2008 (Ministry of Health, 2008), giving patients a greater opportunity to obtain large quantities of sedatives at a time. To prevent sedative dependence/abuse under these circumstances, undergraduate and postgraduate education regarding BZ dependence needs to be strengthened, and training workshops for psychiatrists and other healthcare professionals need to be implemented at the initiative of psychiatric societies.

\subsection{Limitations}

We close this section with a discussion of the four major limitations of this study.

First, the subjects were limited to patients with a drug-related disorder who had consulted or been admitted to a hospital that had an inpatient psychiatric unit, which meant that no such patients who had consulted a psychiatric institution or psychiatric clinic without an inpatient unit were included in this study. As a result, the generalizability of findings to the entire population of drug abusers and addicts in Japan is somewhat limited.

Second, the psychiatric hospitals that participated in the Hospital Survey in 2010, on which this study was based, accounted for only $63.3 \%$ of all hospitals in Japan that have an inpatient psychiatric unit. In addition, only $70.4 \%$ of the potential subjects during the survey period were included in the analyses in this study. Thus, the subjects did not represent the entire population of patients with a drug-related disorder in Japan who were being treated at hospitals with a psychiatric inpatient unit. It should be noted, however, that our survey had an adequate response rate for a survey conducted by the postal method.

Third, the attending psychiatrists at each institution obtained the information that served as the basis of our study during an interview of their patients who had a drug-related disorder. Although the information was collected by means of a structured interview, we cannot rule out the possibility that the criteria for assessment and evaluation of the subjects' response to each question may have varied to some extent from psychiatrist to psychiatrist.

Fourth and finally, because the comparisons between the results obtained in the SRD group and MRD group were not adjusted for age or gender, the clinical characteristics of the SRD patientsidentified in this study may be those of young or female drug abusers or addicts 
rather than being specific to patients with a SRD. In fact, the comorbid psychiatric disorders and self-destructive behaviors found in the SRD patients have been identified as characteristics of females who are drug dependent or drug abusers (Matsumoto et al., 2003). Nonetheless, because the intention of this study was to describe the raw characteristics of SRD patients seen in psychiatric settings, we consciously did not adjust the results for age or gender.

In spite of these limitations, the Hospital Survey is the only inventory survey that has been conducted over time inpatients with drug-related disorders in Japan, and the study reported here is the first to elucidate the clinical characteristics of recent Japanese SRD patients in comparison with MRD patients.

\section{CONCLUSION}

The subjects of this study were 671 patients with a drug-related disorder who consulted or were admitted to a hospital with an inpatient psychiatric unit in Japan in the months of September and October 2010. The attending psychiatrist of each patient interviewed the subject or collected relevant information from the subject's medical records. The data were analyzed to determine the proportion of subjects who considered a sedative (hypnotic or anxiolytic) to be the primarydrug responsible for their drug-related disorder as well as to identify the clinical characteristics of SRD patients compared to those of MRD patients. The results showed that sedative-related disorders increased as a proportion of drug-related disorders as a whole, but the decrease in number of patients with anorganic solvent-related disorder in their background also appears to have had an impact. In addition, the clinical characteristics of SRD patients were considerably different from those of MRD patients in terms of both psychosocial and psychiatric aspects. Moreover, a larger proportion of SRD patients reported a recent episode ofsuicidal behavior, suggesting that they may have overlapped with the group of patients who took an overdose of a psychoactive substance.

In conclusion, to treat sedative-related disorders, there is an urgent need to develop treatment programs focused on the main clinical features of the disorders (i.e., Dependence syndrome and Harmful use) and to make them widely available. From the standpoint of preventing sedative abuse, education regarding $\mathrm{BZ}$ dependence should be emphasized in undergraduate and postgraduate education in medical schools, as well as in training workshops for healthcare professionals conducted by psychiatric societies.

\section{ACKNOWLEDGEMENTS}

This study was based on the results of the Study on the Current Status of Drug Abuse/Dependence and the Challenges to Social Resources of Preventing Relapses of Drug Abuse (principal investigator: Kiyoshi Wada), which was supported by a Health Labour Sciences Research Grant (Research on Regulatory Science of Pharmaceuticals and Medical Devices) from the Ministry of Health, Labour and Welfare of Japan.

We are extremely grateful to the physicians, other medical professionals, and patients at the hospitals in Japan for participating in this Hospital Survey. The original version of this paper, written in Japanese, has already been published on the Seishinshinkeigaku-zasshi (Psychiatria et Neurologia Japonica), vol. 113, 1184-1198, 2011. We gratefully appreciate the editorial board of the Seishinshinkeigaku-zasshi permitting reproduction from the journal. 


\section{REFERENCES}

Cabinets Office (2010). Chapter 1. Current situation of suicides in Japan. The white paper on suicide prevention 22-24.

Griffiths, R.R., \& Weerts, E.M. (1997). Benzodiazepine self-administration in humans and laboratory animals-implications for long-term use and abuse. Psychopharmacology, 134, 11-37,

Hirokawa, S., Matsumoto, T., Katsumata, Y., Kitani, M., Akazawa, M., Takahashi, Y., Kawakami, N., Watanabe, N., Hirayama, M., Kameyama, A., \& Takeshima, T. (2012). Psychosocial and psychiatric characteristics of suicide completers with psychiatric treatment before death: A psychological autopsy study of 76 cases. Psychiatry and Clinical Neurosciences, 66, 292-302.

Khantzian, E.K. (1990). Self-regulation and self-medication factors in alcoholism and the addictions: Similarities and differences. In Galanter, M., Eds.: Recent Developments in Alcoholism, pp.251-277, Plenum, New York.

Kondo, T., Ishizuka, S., \& Shigeta, S. (2010). Keeping the addicts' company for their recovery: Magnanimity of DARC. Gendai-Shisou, 38, 56-79, (in Japanese).

Kondo, T. (2000). Overcoming drug dependence: The program for recovery and rebirth. Kaitakusha, Tokyo, (in Japanese).

Lader, M., \& Petursson, H. (1981). Benzodiazepine derivatives, side effect and dangers. Biological. Psychiatry, 16, 1195-1201.

Linehan, M.M., Shireen, L.R., \& Welch, S.S. (2006). Psychiatric aspects of suicidal behavior: Personality disorders. In Hawton, K., van Heeringen, K., Eds.: The international handbook od suicide and attempted suicide, pp.147-178, John Wiley \& Sons, Chichester,

Lönnqvist, J.K., Henriksson, M.M., Isometsä, E.T., Marttunen, M.J., Heikkinen, M.E., Aro, H.M., \& Kuoppasalmi, K.I. (1995). Mental disorders and suicide prevention. Psychiatry and Clinical Neurosciences, 49, Suppl 1:S111-116,

Mainichi Shinbun: Kokoro wo sukuu: (Saving mind: Wandering for 12 years). Mainichi Shinbun 24/6/2010, morning edition (in Japanese).

Mainichi Shinbun: Kokoro wo sukuu: (Saving mind: Wandering for 12 years). Mainichi Shinbun 27/6/2010, morning edition (in Japanese).

Matsumoto, T., \& Kobayashi, O. (2010). Psychosocial treatment of substance use disorder: Cognitive behavioral therapy for relapse prevention. Psychiatria et Neurologia Japonica (Seishinshinkeigaku Zasshi), 112, 672-676 (in Japanese).

Matsumoto, T., Yamaguchi, A., Kamijo, A., et al. (2003). Eating disorders in female substance use disorders: A preliminary research on the relationship of substances and eating behaviors. SeishinIgaku, 45, 119-127 (in Japanese).

Mental health and welfare in Japan (Wagakuni no Seishin Hoken Fukushi): Chapter 3. Policies for mentally disordered individuals. Mental health and welfare in Japan (The handbook of mental health), 69-111, Tokyo, 2010 (in Japanese).

Ministry of Health, Labour and Welfare of Japan: Medicines of which number of days limited to be prescribed.Official gazette 19/3/2008 (extra edition, No.55) (in Japanese).

Ministry of Health, Labour and Welfare of Japan: Press announcement by the projects team for "Prevention of overdosing: For psychiatric practice not resorting only pharmacotherapy."9/9/2009(in Japanese).

Ministry of Health, Labour and Welfare of Japan: Preventing suicidal behavior by overdose of prescribed psychotropic drugs.Archives 0624-1 of Division of Mental Health and Disability Health, Department of Health and Welfare for Persons with Disabilities, Social Welfare and War Victims' Relief Bureau, 24/6/2010 (in Japanese).

Murasaki, M. (2001). Current situation and scope of psychotropic drugs in japan.: Toward 21th Centuries. Rinsho Seishin-yakuri, 4, 3-27,

Okura, R., Mino, K., \& Kokake, M. (2008). Clinical characteristics of patients with psychotropic drug overdose admitted to the emergency department. Nihon Kyukyu Igakukai Zasshi, 19, 901-913. 
Ozaki, S., Kikuchi, S., \& Wada, K. (2001). Characteristics of patients with hypnotic-related psychiatric disorders in the nationwide mental hospital survey. Psychiatry and Clinical Neurosciences, 55, 205-207.

Ozaki, S., Wada, K., Otsuki, N. (2009). A nationwide survey of drug-related psychiatric illness in hospitals with psychiatric inpatient units. The Research Report of 2008 Health Labour Sciences Research Grant (Research on Regulatory Science of Pharmaceuticals and Medical Devices) from the Ministry of Health, Labour and Welfare of Japan, 87-134 (in Japanese).

Ribeiro, C.S., Azevedo, R.C., Silva, V.E., \& Botega, N.J. (2007). Chronic use of diazepam in primary healthcare centers: user profile and usage pattern. Sao Paulo Medical Journal, 125, 270-274,

Rickels, K., Case, W.G., Downing, R.W., \& Winokur, A. (1983). Long-term diazepam therapy and clinical outcome. JAMA, 250, 767-771.

Tajima, O. (2001). Rethinking prescription of benzodiazepines. Rinsho SeishinIgaku, 30, 1065-1069 (in Japanese).

Takei, A., Mera, K., Miyazaki, K., et al. (2007). Clinical Characteristics of Drug Overdose Patients: A report from the emergency room of a general hospital. Japanese Journal of General Hospital Psychiatry, 19, 211-219, (in Japanese).

Tennant, F.S. Jr., \& Pumphrey, E.A. (1984). Benzodiazepine dependence of several years duration: clinical profile and therapeutic benefits. NIDA Research Monograph, 55, 211-216,

The Royal College of Psychiatrists (1997. CR59. Benzodiazepines: risks, benefits and dependence A reevaluation. Council Report CR59 January 1997. Royal College of Psychiatrists, London,

Wada, K., \& Fukui, S. (1991). What is drug dependence caused from. Seishin Igaku, 33, 633-642.

Wada, K., Kobori, E., Shimane, T., et al. (2011). A nationwide survey of actual condition of drinking, smoking, and drug abuse among Japanese junior highschool students (2010): The Research Report of 2010 Health Labour Sciences Research Grant (Research on Regulatory Science of Pharmaceuticals and Medical Devices) from the Ministry of Health, Labour and Welfare of Japan, 89-115.

Wada, K. (2011). The history and current state of drug abuse in Japan. Annals of the New York Academy of Sciences, 1216, 62-72.

Wunsch, M.J., Boyd, C., \& McMasters, M.G. (2009). Chapter 33. Nonmedical use of prescription medications. In Ries, R.K., Fiellin, D.A., Miller, S.C., et al. (eds.) Principles of addiction medicine, fourth edition, pp. 453-463, Wolters Kluwer/Lippincott Williams \& Wilkins, Philadelphia. 